\title{
An operator splitting scheme for the stream-function formulation of unsteady Navier-Stokes equations
}

\author{
C. I. Christov*, $\dagger$ and X.-H. Tang \\ Department of Mathematics, University of Louisiana at Lafayette, LA 70504-1010, U.S.A.
}

\begin{abstract}
SUMMARY
A fictitious time is introduced into the unsteady equation of the stream function rendering it into a higher-order ultra-parabolic equation. The convergence with respect to the fictitious time (we call the latter 'internal iterations') allows one to obtain fully implicit nonlinear scheme in full time steps for the physical-time variable. For particular choice of the artificial time increment, the scheme in full time steps is of second-order of approximation. For the solution of the internal iteration, a fractional-step scheme is proposed based on the splitting of the combination of the Laplace, bi-harmonic and advection operators. A judicious choice for the time staggering of the different parts of the nonlinear advective terms allows us to prove that the internal iterations are unconditionally stable and convergent. We assess the number of operations needed per time step and show computational effectiveness of the proposed scheme. We prove that when the internal iterations converge, the scheme is second-order in physical time and space, nonlinear, implicit and absolutely stable. The performance of the scheme is demonstrated for the flow created by oscillatory motion of the lid of a square cavity. All theoretical findings are demonstrated practically. Copyright (c) 2006 John Wiley \& Sons, Ltd.
\end{abstract}

Received 25 January 2006; Revised 18 April 2006; Accepted 19 April 2006

KEY WORDS: unsteady Navier-Stokes equations; operator splitting; implicit schemes

\section{INTRODUCTION}

The system of Navier-Stokes $(\mathrm{N}-\mathrm{S})$ equations describes the motion of incompressible viscous liquid. The coupling of $\mathrm{N}-\mathrm{S}$ system comes through the nonlinear terms, the continuity equation, and the boundary conditions. In numerical solutions of the incompressible $\mathrm{N}-\mathrm{S}$, a serious difficulty arises from the determination of the pressure field. The unknown pressure function provides the degree of freedom needed to accommodate the incompressibility constraint and requires an implicit

\footnotetext{
*Correspondence to: C. I. Christov, Department of Mathematics, University of Louisiana at Lafayette, LA 705041010, U.S.A.

${ }^{\dagger}$ E-mail: christov@louisiana.edu
} 
determination. This means that the incompressible $\mathrm{N}-\mathrm{S}$ equations are not a system of CauchyKowalevska type [1] and this aspect can be considered to be their most distinctive feature. Quite naturally, for the implicit pressure function no boundary conditions (b.c.) are to be prescribed at the rigid boundaries. This is still another formidable obstacle on the way of constructing fully implicit schemes. In 2D, the pressure can be eliminated from the equations by means of stream function $\psi$ deriving a fourth-order equation for the latter.

In order to reduce the fourth-order equation to a set of second-order elliptic equations, a vorticity function $\omega=-\nabla^{2} \psi$ is introduced in many numerical works. The idea to add time derivative of the stream function in the above equation was first suggested in Reference [2] and the method was called 'method of false transients'. The good news about this method was that the system of equation became parabolic and amenable to iterative approaches. Naturally, the transients do not follow the real time dependence of the solution and thus it is just an efficient iterative method for stationary solutions. It was shown [3] that if the artificial time derivative is multiplied by a small parameter, then the solution approximates the solution of the unsteady problem within the first order of that small parameter.

Although apparently more tractable, the $\psi-\omega$ formulation is inherently explicit, because of the lack of boundary condition for the vorticity function $\omega$. This fact is a straightforward corollary from the implicit nature of the pressure function. The vorticity is also an implicit function for which no boundary condition can be specified except for the special cases of prescribed stress function on a free surface or interface. There is another problem with this semi-explicit approach connected with the rigorous demonstration of the approximation since it depends on the iterative process.

The implicit nature of the pressure function (or the vorticity function) requires a special care for stability of algorithms since the explicit decoupling of the boundary conditions (descendant of the so-called Thom's condition) imposes significant limitations on the time increment [2-5] for stability. The literature offers various approaches to the problems of the iterative decoupling the system. The continuity equation is decoupled by means of Chorin's method of fractional steps (see References [6-8]) in which the velocity field is predicted in the first half-time step and then the pressure is adjusted in the second half-time step so that the momentum equations are projected on approximately divergence-free vector field. A comprehensive account of the progress recently made along these lines can be found in References $[9,10]$ where the $3 \mathrm{D}$ problem is also treated. This kind of methods are called semi-explicit. One is referred to the comprehensive review [11] for the literature on the subject.

In the quest for implicit difference scheme, one can use the stream function formulation $(\psi$ formulation) without introducing the vorticity function. In doing so, however, one is faced with a spatially fourth-order (bi-harmonic) problem. While it is now completely natural to pose two b.c. for $\psi$, the price to be paid is the increased order of the boundary value problem. To the limit of our knowledge, the first numerical solution of $\mathrm{N}-\mathrm{S}$ with bi-harmonic equation for the stream function is attempted in Reference [12] using relaxation method. The first implicit stream-function scheme is presented in Reference [13]. In the present paper, we tackle the problem of creating absolutely stable implicit scheme for the unsteady $\psi$ formulation. For the time being we concern ourselves with the $2 \mathrm{D}$ case, but the experience can be translated to $3 \mathrm{D}$ without ideological adjustments in the framework of one of the vector-potential formulations.

A fully implicit scheme in more than one spatial dimensions requires inversion of very large matrices and is resource intensive. One of the most efficient approaches to reducing the computational time without compromising the stability of the scheme is the method of operator splitting 
that was introduced in the late 1950s, in References [14-16] (for Russian literature references see Reference [17]). Generically speaking, this is a fractional step method but in order to avoid ambiguity of the terminology we will call it 'operator splitting' method meaning that a time is broken into two fractional 'half-time' steps in which 1D operators are inverted. The application of the splitting method to $\mathrm{N}-\mathrm{S}$ equations in $\psi$-formulation is not straightforward because of the fact that they are not a Cauchy-Kowalevska system and there is no time derivative of the stream function. What is present in the equation is the term $\nabla^{2} \psi_{t}$ which cannot serve as a basis for splitting.

For stationary flows where a mixed time-spatial derivative $\nabla^{2} \psi_{t}$ is not present one can add artificial time and treat the problem as higher-order diffusion equation in which the spatial derivatives are represented by the bi-harmonic operator. For bi-harmonic problems, the first application of the operator splitting method was outlined in Reference [18]. Application to stationary N-S equation of the splitting method with artificial time is implemented in Reference [19], whereas featuring example used is the well known lid-driven cavity flow. The artificial-time approach with splitting for solving stationary $\mathrm{N}-\mathrm{S}$ equations was developed further for the primitive variables and highly accurate and stable results were obtained for the lid-driven flow [20] and the flow behind a backward-facing step [21].

In order to be able to use the operator splitting method one has to have a time derivative in the equation, i.e. the equation has to be evolution equation of Cauchy-Kowalevska type. If the original physical formulation does not feature such a time derivative, the latter can be added as an artificial time and then to consider the artificial-time evolution as an iterative process to solve the real physical problem within one single physical time step. This idea was first proposed in Reference [22]. Some preliminary calculations for the lid-driven flow showed the effectiveness of the method. Recently, the operator-splitting algorithm was generalized to include an equation for the transport of passive scalar and applied to the complex thermoconvective flow in vertical slot under gravity modulations (so-called $g$-jitter flow) [23].

The main advantage of the approach of the present paper is that the boundary conditions are treated in strictly implicit fashion in the way they are posed-as two conditions on the stream function. It is of separate theoretical interest to investigate also the specific problems when operator splitting methods are used for complex operators involving both Laplacian and bi-harmonic operators.

Here we concern ourselves with the mathematical problems connected with the new splitting scheme. We prove the absolute stability and convergence of the internal iterations and the stability of the scheme for the physical time. Problems connected with the approximation and the consistency of the scheme are also clarified.

\section{GOVERNING EQUATIONS}

We consider the 2D N-S equation for incompressible unsteady flows in terms of stream function $\psi$ which has the form:

$$
\frac{\partial \Delta \psi}{\partial t}+\operatorname{Re}\left(\frac{\partial \psi}{\partial y} \frac{\partial \Delta \psi}{\partial x}-\frac{\partial \psi}{\partial x} \frac{\partial \Delta \psi}{\partial y}\right)-\Delta^{2} \psi=0
$$


In dimensionless variables, we may restrict our attention to the closed rectangle $\bar{D}=\{(x, y) \mid 0 \leqslant x \leqslant 1$, $0 \leqslant y \leqslant 1\}$ and $R e=U L / v$ defined as the Reynolds number based on the characteristic length scale $L$ and velocity $U$, where we let $D$ denote the open rectangle region. The geometrical simplicity of the rectangle $\bar{D}$ makes it easier to exemplify the splitting method described in later sections. The more complicated region requires more work on coordinate splitting near the boundary [17, 24], which we will leave for future research. If we have in mind a driven cavity flow, then the boundary conditions on $\partial D$ are the following:

$$
\begin{aligned}
& \psi=\frac{\partial \psi}{\partial x}=0 \quad \text { for } x=0 \text { and } 1 \\
& \psi=\frac{\partial \psi}{\partial y}=0 \quad \text { for } y=0 \\
& \psi=0 \frac{\partial \psi}{\partial y}=g(x, t) \quad \text { for } y=1, \quad 0 \leqslant x \leqslant 1 \text { and } t>0
\end{aligned}
$$

with $g(x, t)$ being the distribution of the longitudinal velocity at the plane $y=1$. Generally, other combinations of boundary conditions can also be treated without much difficulty by the numerical scheme in our paper. We impose the initial condition for the stream function as follows:

$$
\psi(x, y, 0)=0 \quad \text { for all }(x, y) \in \bar{D}
$$

since in the initial moment the fluid is at rest and the flow is created by the sudden appearance of velocity distribution at the top boundary $y=1$.

\section{DIFFERENCE OPERATORS}

In order not to obscure the main ideas, we take a uniform grid on the closed rectangle $\bar{D}$. Let us divide each of the intervals $0 \leqslant x \leqslant 1$ and $0 \leqslant y \leqslant 1$ into $M$ equal subintervals $x_{i} \leqslant x \leqslant x_{i+1}$ and $N$ subintervals $y_{j} \leqslant y \leqslant y_{j+1}$ of lengths $h_{x}=x_{i+1}-x_{i}$ and $h_{y}=y_{j+1}-y_{j}$, respectively. Therefore:

$$
\psi_{i j}^{n}=\psi\left[x_{i}=i h_{x}, y_{j}=j h_{y}, t_{n}=(n-1) \tau\right]
$$

for $i=0,1, \ldots, M ; j=0,1, \ldots, N$; and $n=1,2, \ldots$ Here $\tau$ is the time increment (time step length). We also observe that Equation (2) contains first-order spacial derivatives posed on the sides of the rectangular domain $\bar{D}$. In order to obtain second-order accuracy difference approximation, the solution of the problem (which is assumed to be sufficiently smooth) is extended to the additional intervals of length $h_{x}$ adjacent to the left and right boundaries of the domain, and intervals of length $h_{y}$ adjacent to the top and bottom of the boundary. This means that we consider an extended grid overlapping the four boundaries:

$$
\begin{aligned}
& x_{-1}=-h_{x}, \quad x_{0}=0, \quad x_{i}=i h_{x}, \quad x_{M}=1, \quad x_{M+1}=1+h_{x} \\
& y_{-1}=-h_{y}, \quad y_{0}=0, \quad y_{j}=j h_{y}, \quad y_{N}=1, \quad y_{N+1}=1+h_{y}
\end{aligned}
$$

Next, let us introduce the forward and backward difference operators as follows:

$$
\left(\delta_{x}^{+} \psi\right)_{i j}=\frac{1}{h_{x}}\left(\psi_{i+1, j}-\psi_{i j}\right), \quad\left(\delta_{x}^{-} \psi\right)_{i j}=\frac{1}{h_{x}}\left(\psi_{i, j}-\psi_{i-1, j}\right)
$$




$$
\left(\delta_{y}^{+} \psi\right)_{i j}=\frac{1}{h_{y}}\left(\psi_{i, j+1}-\psi_{i j}\right), \quad\left(\delta_{y}^{-} \psi\right)_{i j}=\frac{1}{h_{y}}\left(\psi_{i, j}-\psi_{i, j-1}\right)
$$

Then we have for the central difference operators

$$
\begin{aligned}
& \left(\Lambda_{x^{2}} \psi\right)_{i j}=\delta_{x}^{+} \delta_{x}^{-}=\frac{1}{h_{x}^{2}}\left(\psi_{i+1, j}-2 \psi_{i j}+\psi_{i-1, j}\right) \\
& \left(\Lambda_{y^{2}} \psi\right)_{i j}=\delta_{y}^{+} \delta_{y}^{-}=\frac{1}{h_{y}^{2}}\left(\psi_{i, j+1}-2 \psi_{i j}+\psi_{i, j-1}\right)
\end{aligned}
$$

We use the notation $(\Lambda \phi)_{i j}$ to denote the implementation of a difference operator $\Lambda$ on the function $\phi$ at the grid point $\left(i h_{x}, j h_{y}\right)$. Then we can write for convenience

$$
-\Delta_{h}=-\delta_{x}^{+} \delta_{x}^{-}-\delta_{y}^{+} \delta_{y}^{-}=-\Lambda_{x^{2}}-\Lambda_{y^{2}}
$$

The central difference operators $\Lambda_{x^{4}}, \Lambda_{y^{4}}$, and $\Lambda_{x^{2}} \Lambda_{y^{2}}$ approximate the fourth-order derivatives $\partial^{4} / \partial x^{4}, \partial^{4} / \partial y^{4}$ and $\partial^{4} / \partial^{2} x \partial^{2} y$, namely

$$
\begin{gathered}
\left(\Lambda_{x^{4}} \psi\right)_{i j}=\left(\Lambda_{x^{2}} \Lambda_{x^{2}} \psi\right)_{i j}=\frac{1}{h_{x}^{4}}\left(\psi_{i+2, j}-4 \psi_{i+1, j}+6 \psi_{i j}-4 \psi_{i-1, j}+\psi_{i-2, j}\right) \\
\left(\Lambda_{y^{4}} \psi\right)_{i j}=\left(\Lambda_{y^{2}} \Lambda_{y^{2}} \psi\right)_{i j}=\frac{1}{h_{y}^{4}}\left(\psi_{i, j+2}-4 \psi_{i, j+1}+6 \psi_{i j}-4 \psi_{i, j-1}+\psi_{i, j-2}\right) \\
\left(\Lambda_{x^{2}} \Lambda_{y^{2}} \psi\right)_{i j}=\frac{1}{h_{x}^{2} h_{y}^{2}}\left[\psi_{i+1, j+1}-2 \psi_{i, j+1}+\psi_{i-1, j+1}-2\left(\psi_{i+1, j}-2 \psi_{i, j}+\psi_{i-1, j}\right)\right. \\
\left.+\psi_{i+1, j-1}-2 \psi_{i, j-1}+\psi_{i-1, j-1}\right] .
\end{gathered}
$$

Since $\Lambda_{x^{2}}$ and $\Lambda_{y^{2}}$ commute, we have $\Lambda_{x^{2}} \Lambda_{y^{2}}=\Lambda_{y^{2}} \Lambda_{x^{2}}$ which is very useful for the factorization of operators in the splitting scheme as presented in a following section.

The following typical notations are used throughout:

$$
\begin{gathered}
(\hat{\psi}, \hat{\phi})=(\hat{\phi}, \hat{\psi})=h_{x} h_{y} \sum_{i=1}^{M-1} \sum_{j=1}^{N-1} \hat{\psi}_{i j} \hat{\phi}_{i j} \\
(\Lambda \hat{\psi}, \hat{\psi})=h_{x} h_{y} \sum_{i=1}^{M-1} \sum_{j=1}^{N-1}(\Lambda \hat{\psi})_{i j} \hat{\psi}_{i j} \\
\|\hat{\psi}\|=(\hat{\psi}, \hat{\psi})^{1 / 2}=\left(h_{x} h_{y} \sum_{i=1}^{M-1} \sum_{j=1}^{N-1} \hat{\psi}_{i j}^{2}\right)^{1 / 2}
\end{gathered}
$$

where $\hat{\psi}$ and $\hat{\phi}$ are grid functions defined on the same grid Equation (4) along with $\Lambda$ being a difference operator acting upon the grid function $\left(\hat{\psi}_{i j}\right)$.

We assume that the stream function has continuous derivatives up to eighth order. At this point is enough to have sixth-order continuous derivatives but when dealing with the pointwise error 
of the splitting scheme, we will have to assume the higher smoothness order. So, for sufficiently smooth functions, we make use of some general results (see, e.g. Reference [25]):

\section{Theorem 1}

Consider the advection operator $A \stackrel{\text { def }}{=} a(x, y)(\partial / \partial x)+b(x, y)(\partial / \partial y)$. If $a=a(x, y, t)$ and $b=b(x$, $y, t)$ satisfy the continuity equation $(\partial a / \partial x)+(\partial b / \partial y)=0$ for $(x, y) \in D$ and $t \geqslant 0$, then with $t \geqslant 0$ fixed, $(A \chi, \chi)=0$ for $\chi=\chi(x, y, t)$ defined on $\bar{D} \times\{t \geqslant 0\}$ if one of the following assumptions holds:

(i) $\chi$, as well as $a$ and $b$, satisfy periodic boundary conditions on $\partial D$, with identical values on the opposite sides of the rectangle.

(ii) $\chi$ vanishes on $\partial D$ or $a$ and $b$ are both zeros on $\partial D$ for $t \geqslant 0$.

\section{Theorem 2}

If $\hat{\phi}$ is the grid function approximating $\chi$, it is possible to decompose $A$ into two operators with respect to different spacial derivatives and construct corresponding second-order difference operators $A_{x}^{h}$ and $A_{y}^{h}$ such that $A^{h}=A_{x}^{h}+A_{y}^{h}$ and $\left(A_{x}^{h} \hat{\phi}, \hat{\phi}\right)=\left(A_{y}^{h} \hat{\phi}, \hat{\phi}\right)=0$, where $A_{x}^{h}$ and $A_{y}^{h}$ are defined as

$$
\begin{aligned}
& \left(A_{x}^{h} \hat{\phi}\right)_{i j}=\frac{1}{2 h_{x}}\left(a_{i+(1 / 2), j} \hat{\phi}_{i+1, j}-a_{i-(1 / 2), j} \hat{\phi}_{i-1, j}\right) \\
& \left(A_{y}^{h} \hat{\phi}\right)_{i j}=\frac{1}{2 h_{y}}\left(b_{i, j+(1 / 2)} \hat{\phi}_{i, j+1}-b_{i, j-(1 / 2)} \hat{\phi}_{i, j-1}\right)
\end{aligned}
$$

where

$$
\begin{array}{ll}
a_{i+(1 / 2), j}=\frac{a_{i+1, j}+a_{i, j}}{2}, & a_{i-(1 / 2), j}=\frac{a_{i, j}+a_{i-1, j}}{2} \\
b_{i, j+(1 / 2)}=\frac{b_{i, j+1}+b_{i, j}}{2}, & b_{i, j-(1 / 2)}=\frac{b_{i, j}+b_{i, j-1}}{2}
\end{array}
$$

Following a coinage from [25], we call this kind of difference operators antisymmetric (or conservative).

The approximation of the nonlinear terms is the other formidable obstacle on the way of constructing of a successful numerical schemes for $\mathrm{N}-\mathrm{S}$ equations. It is well known that the symmetric central differences for the advective terms can lead to instability. A successful remedy to this difficulty is to use upwind differences but then the spatial approximation is reduced to first order, namely $O(h R e)$. This is hardly acceptable for moderate and large Reynolds numbers. Another approach is to use conservative approximation of the nonlinear term as suggested in Reference [26] for inviscid problems. A conservative approximation of the nonlinear term secures that the energy would not increase in time which allows to make the scheme stable. In addition, a conservative central-difference approximation does not introduce scheme diffusion (viscosity). Naturally, the conservative approximation does not remove the scheme dispersion and hence one cannot secure monotonicity of the scheme, but the effects connected with the scheme dispersion are of the second-order $O\left(h^{2}\right)$ and result mostly in phase errors.

The above arguments define our choice to use in the present work a scheme that is adaptation of the classical Arakawa scheme [26] to the implicit operator splitting. 
Usually, the advective nonlinear terms can be written in the form $A \omega=a(x, y, t)(\partial \omega / \partial x)+$ $b(x, y, t)(\partial \omega / \partial y)$, where $a$ and $b$ can be generally functions of the transported scalar $\omega$, but at a given time stage they are considered as known functions of the spatial coordinates and time (the socalled concept of 'frozen coefficients'). The analytical properties and numerical treatments of the operator $A$ are exhaustively discussed in Reference [25] for the case when $(\partial a / \partial x)+(\partial b / \partial y)=0$.

In $\psi-\omega$ variables, $a$ and $b$ are, respectively, the velocity components $\partial \psi / \partial y$ and $-\partial \psi / \partial x$, and $\omega=-\Delta \psi$ is considered as a scalar substance transported by the flow. In the previous authors' works, this kind of interpretation of the advective terms was widely used to achieve different level of refinement of the approximation. In this paper, we acknowledge better the requirements for strictly conservative splitting schemes for $\psi$-formulation. To this end, we observe that similarly to velocity components, the functions

$$
P=-\frac{\partial \Delta \psi}{\partial y} \quad \text { and } \quad Q=\frac{\partial \Delta \psi}{\partial x}
$$

satisfy a continuity-like equation $(\partial P / \partial x)+(\partial Q / \partial y)=0$ on $\bar{D} \times\{t \geqslant 0\}$. Then we rewrite the convection terms as follows:

$$
A \psi=P \frac{\partial \psi}{\partial x}+Q \frac{\partial \psi}{\partial y}
$$

hinting at the idea that now the vorticity can be thought of as a known function while approximating the advective terms. Accordingly, the derivatives of the stream function are treated implicitly. In order to improve the order of approximation in time, one can use the the standard extrapolation formula

$$
\hat{\psi}_{i j}^{n+(1 / 2)} \stackrel{\text { def }}{=} \frac{3}{2} \psi_{i j}^{n}-\frac{1}{2} \psi_{i j}^{n-1}=\psi_{i j}^{n+(1 / 2)}+O\left(\tau^{2}\right)
$$

when evaluating the coefficients $P$ and $Q$. We achieve second-order approximation in time on the expense of using a multilevel scheme. However, this is not a real shortcoming, because one can always set $\psi^{1 / 2}=\psi^{0}$ introducing an error $O(\tau)$ in the initial condition. Even this error can easily be corrected if needed by some kind of corrector scheme used only for the first time step. Thus

$$
\begin{aligned}
P_{i j}^{n+(1 / 2)} & =-\frac{1}{2 h_{y}}\left[\left(\Delta_{h} \hat{\psi}^{n+(1 / 2)}\right)_{i, j+1}-\left(\Delta_{h} \hat{\psi}^{n+(1 / 2)}\right)_{i, j-1}\right] \\
& =-\frac{\partial \Delta \psi}{\partial y}\left(i h_{x}, j h_{y}, \tau(n+1 / 2)\right)+O\left(\tau^{2}+h_{x}^{2}+h_{y}^{2}\right)
\end{aligned}
$$

for $i=0,1, \ldots, M ; j=1, \ldots, N-1$.

$$
\begin{aligned}
Q_{i j}^{n+(1 / 2)} & =\frac{1}{2 h_{x}}\left[\left(\Delta_{h} \hat{\psi}^{n+(1 / 2)}\right)_{i+1, j}-\left(\Delta_{h} \hat{\psi}^{n+(1 / 2)}\right)_{i-1, j}\right] \\
& =\frac{\partial \Delta \psi}{\partial x}\left(i h_{x}, j h_{y}, \tau(n+1 / 2)\right)+O\left(\tau^{2}+h_{x}^{2}+h_{y}^{2}\right)
\end{aligned}
$$

for $i=1, \ldots, M-1 ; j=0,1, \ldots, N$. 
We construct antisymmetric difference operators, $C_{x}^{n+(1 / 2)}$ and $C_{y}^{n+(1 / 2)}$ along $x$ and $y$ directions, respectively, acting upon $\psi$ in the middle time stage $\left(n+\frac{1}{2}\right)$ in order to secure second-order approximation with respect to time. For the sake of demonstrating the approximation, we use the notation

$$
\psi_{i j}^{n+(1 / 2)} \stackrel{\text { def }}{=} \frac{1}{2}\left(\psi_{i j}^{n}+\psi_{i j}^{n+1}\right)
$$

but in the actual implementation of the algorithm, the values of the grid function $\psi^{n+1}$ are considered unknown, while the values of $\psi^{n}$ are treated as known. Acknowledging the fact that the stream function $\psi$ vanishes on the boundary, we can use Theorems 1 and 2 to identify the antisymmetric advective operators as follows:

$$
\begin{aligned}
& \left(C_{x}^{n+(1 / 2)} \psi^{n+(1 / 2)}\right)_{i j}=\frac{1}{2 h_{x}}\left(P_{i+(1 / 2), j}^{n+(1 / 2)} \psi_{i+1, j}^{n+(1 / 2)}-P_{i-(1 / 2), j}^{n+(1 / 2)} \psi_{i-1, j}^{n+(1 / 2)}\right) \\
& \left(C_{y}^{n+(1 / 2)} \psi^{n+(1 / 2)}\right)_{i j}=\frac{1}{2 h_{y}}\left(Q_{i, j+(1 / 2)}^{n+(1 / 2)} \psi_{i, j+1}^{n+(1 / 2)}-Q_{i, j-(1 / 2)}^{n+(1 / 2)} \psi_{i, j-1}^{n+(1 / 2)}\right)
\end{aligned}
$$

for $i=1, \ldots, M-1, j=1, \ldots, N-1$. Here $P$ and $Q$ are evaluated from the following finite differences with the second-order accuracy $O\left(h_{x}^{2}+h_{y}^{2}+\tau^{2}\right)$, namely:

$$
\begin{array}{cc}
P_{i+(1 / 2), j}^{n+(1 / 2)}=\frac{P_{i+1, j}^{n+(1 / 2)}+P_{i, j}^{n+(1 / 2)}}{2}, & P_{i-(1 / 2), j}^{n+(1 / 2)}=\frac{P_{i, j}^{n+(1 / 2)}+P_{i-1, j}^{n+(1 / 2)}}{2} \\
Q_{i, j+(1 / 2)}^{n+(1 / 2)}=\frac{Q_{i, j+1}^{n+(1 / 2)}+Q_{i, j}^{n+(1 / 2)}}{2}, & Q_{i, j-(1 / 2)}^{n+(1 / 2)}=\frac{Q_{i, j}^{n+(1 / 2)}+Q_{i, j-1}^{n+(1 / 2)}}{2}
\end{array}
$$

One should be noted, that the last formulas are essential in constructing the conservative approximation. One can compute the values of $P$ and $Q$ at the middle lines $i \pm \frac{1}{2}$ and $j \pm \frac{1}{2}$ using some different approximations of equalities (15), (16), but then it will not be possible to make the approximation conservative.

Due to Theorem 2 we have that the operators in Equation (18) are anti-symmetric (or conservative), namely

$$
\left(C_{x}^{n+(1 / 2)} \psi, \psi\right)=0, \quad\left(C_{y}^{n+(1 / 2)} \psi, \psi\right)=0, \quad \psi \in \Phi
$$

\section{NONHOMOGENEOUS BOUNDARY CONDITION AND DIFFERENCE SCHEME}

Equations (1) and (2) pose a boundary value problem comprising a homogeneous partial differential equation for stream function $\psi$ and nonhomogeneous boundary conditions. The outline of the ideas of this section is the following: in order to apply Fourier analysis of the numerical problem, we have to eliminate the nonhomogeneous boundary conditions by using the difference analogs of the boundary conditions, the accuracy of which matches that of our final difference equations. Then we obtain a nonhomogeneous system of difference equations by moving all nonhomogeneous boundary conditions to the right-hand side of the system.

By the definitions of the difference operators $C_{x}^{n+(1 / 2)}$ and $C_{y}^{n+(1 / 2)}$, Equations (17)-(19), we are able to give the difference approximation for Equation (1) based on the Crank-Nicholson scheme 
the form:

$$
\begin{aligned}
& \frac{\left(\Delta_{h}\left(\psi^{n+1}-\psi^{n}\right)\right)_{i j}}{\tau}+\left(\left[\operatorname{Re}\left(C_{x}^{n+(1 / 2)}+C_{y}^{n+(1 / 2)}\right)\right.\right. \\
& \left.\left.-\left(\Lambda_{x^{4}}+\Lambda_{y^{4}}+2 \Lambda_{x^{2}} \Lambda_{y^{2}}\right)\right] \frac{\psi^{n+1}+\psi^{n}}{2}\right)_{i j}=F_{i j}^{n+(1 / 2)}
\end{aligned}
$$

for $i=1, \ldots, M-1$ and $j=1, \ldots, N-1$.

From the previous section, it is easy to verify that Equation (21) is of second-order of accuracy both in time and space. Therefore we need to construct difference analogs of the boundary conditions with the accuracy of the same order at every time step $t=n \tau$, which are given as follows:

$$
\begin{gathered}
\psi_{0, j}^{n}=\psi_{M, j}^{n}=0 \\
\psi_{i, 0}^{n}=\psi_{i, N}^{n}=0 \\
\psi_{1, j}^{n}-\psi_{-1, j}^{n}=0, \quad \psi_{M+1, j}^{n}-\psi_{M-1, j}^{n}=0 \\
\psi_{i, 1}^{n}-\psi_{i,-1}^{n}=0, \quad \psi_{i, N+1}^{n}=\psi_{i, N-1}^{n}+2 h_{y} g_{i}^{n}
\end{gathered}
$$

for $i=1, \ldots, M-1$ and $j=1, \ldots, N-1$ with $g_{i}^{n}=g(i h, n \tau)$.

Now, we substitute Equation (22) into Equation (21) and then move all newly generated inhomogeneous terms to right-hand side of the system of difference equations. Here $F_{i j}$ is the nonhomogeneous term of the system and due to the above mentioned b.c., has the form:

$$
F_{i j}^{n+(1 / 2)}= \begin{cases}\frac{1}{h_{y}^{3}}\left(g_{i}^{n+1}+g_{i}^{n}\right) & \text { if } j=N-1 \text { and } i=1, \ldots, M-1 \\ 0 & \text { otherwise }\end{cases}
$$

but now the boundary conditions are homogeneous at every time step $t=n \tau$ :

$$
\begin{aligned}
& \psi_{0, j}^{n}=\psi_{M, j}^{n}=0 \\
& \psi_{i, 0}^{n}=\psi_{i, N}^{n}=0 \\
& \psi_{1, j}^{n}=\psi_{-1, j}^{n}, \quad \psi_{M+1, j}^{n}=\psi_{M-1, j}^{n} \\
& \psi_{i, 1}^{n}=\psi_{i,-1}^{n}, \quad \psi_{i, N+1}^{n}=\psi_{i, N-1}^{n}
\end{aligned}
$$

for $i=1, \ldots, M-1$ and $j=1, \ldots, N-1$.

An important note is due here about the possible discontinuity in boundary conditions. The strict results proved in the present paper and in most of the papers in the literature are valid for boundary conditions that are smooth for the normal derivatives. For instance, the lid-driven cavity flow which is the benchmark for every new algorithm proposed, has discontinuous normal derivative at the upper corners of the cavity where the lid touches the side walls. In fact the derivative (the respective velocity component) is supposed to jump from zero to one in an infinitesimal neighborhood of the corner point. This means that the quantities $g$ are of unit order in the points inside the regions 
that are adjacent to the corner points. Clearly, this will destroy the local approximation of the scheme, but it does nothing more harmful than that. This means that the global estimate of the error remains of second-order $O\left(h^{2}\right)$ and the contribution of a small bunch of points near the corners is negligible. The practical computations (as presented in numerous paper in the literature, as well as in the last section of the present work) do show that this is the case. Naturally if one assumes that the velocity in the lid plane continuously increase from zero to one in the point next to the corner, one can still consider the function $g$ as continuous but with very large derivative. Then one arrives at the same conclusion about the overall approximation. Clearly, when the b.c. is continuous, then it is easily shown that the above quantities $g$ from Equation (23) are of order $O(h)$ and this secures the pointwise second order of the approximation. This problem is common for all known from the literature approaches and its deep investigation goes beyond the scope of present work.

\section{POSITIVITY OF THE DIFFERENCE OPERATORS}

Before we show that scheme Equations (21), (24) is absolutely stable, we consider the issues connected with the positivity of the operators involved in the scheme. For convenience, we use $\left(\psi_{i j}\right) \in \Phi$, to denote the grid function in our computations that satisfy the homogeneous boundary condition Equation (24). Note that the grid where space $\Phi$ is originally defined contains $(M-1) \times(N-1)$ internal points. Since the boundary conditions Equation (24) provide an one-toone correspondence between the functions defined on the internal grid and the functions defined on the extended grid, then $\Phi$ is a space of $(M+3) \times(N+3)$ matrices.

Theorem 3 (Smarskii [27])

$-\Delta_{h}$ is positive definite and self-adjoint, i.e.

$$
\begin{gathered}
\left(-\Delta_{h} \psi, \psi\right)=h_{x} h_{y} \sum_{i=1}^{M} \sum_{j=1}^{N}\left[\left(\delta_{x}^{-} \psi\right)_{i j}^{2}+\left(\delta_{y}^{-} \psi\right)_{i j}^{2}\right]>0 \\
\left(-\Delta_{h} \psi, \psi^{*}\right)=\left(\psi,-\Delta_{h} \psi^{*}\right)=h_{x} h_{y} \sum_{i=1}^{M} \sum_{j=1}^{N}\left[\left(\delta_{x}^{-} \psi\right)_{i j}\left(\delta_{x}^{-} \psi^{*}\right)_{i j}+\left(\delta_{y}^{-} \psi\right)_{i j}\left(\delta_{y}^{-} \psi^{*}\right)_{i j}\right]
\end{gathered}
$$

provided that $\psi$ and $\psi^{*} \in \Phi$ and $\psi$ is non-zero grid function (see, e.g. [27]).

We prove the following theorems to show the positivity of the operators $\Lambda_{x^{4}}, \Lambda_{y^{4}}$ and $\Lambda_{x^{2}} \Lambda_{y^{2}}$.

Theorem 4

If the operators are defined on the domain $\Phi$, then

(i) $\Lambda_{x^{4}}$ and $\Lambda_{y^{4}}$ are positive definite,

(ii) $\Lambda_{x^{2}} \Lambda_{y^{2}}$ is also positive definite.

Proof

(i) At first we show $\left(\Lambda_{x^{4}} \psi, \psi\right)>0$ provided $\psi \in \Phi$ is not zero vector. We may fix the index $j$ and investigate the inner product along each $x$ grid line. For the sake of brevity we can omit the index 
$j$ in the following equations:

$$
\begin{aligned}
h_{x}^{4} \sum_{i=1}^{M-1}\left(\Lambda_{x^{4}} \psi\right)_{i j} \psi_{i j} & =\sum_{i=1}^{M-1}\left(6 \psi_{i}^{2}-4 \psi_{i} \psi_{i-1}-4 \psi_{i} \psi_{i+1}+\psi_{i} \psi_{i-2}+\psi_{i} \psi_{i+2}\right) \\
h_{x}^{4} \sum_{i=1}^{M-1}\left(\delta_{x}^{+} \delta_{x}^{-} \psi\right)_{i j}^{2} & =\sum_{i=1}^{M-1}\left(\psi_{i+1}-2 \psi_{i}+\psi_{i-1}\right)^{2} \\
& =\sum_{i=1}^{M-1}\left(\psi_{i-1}^{2}+\psi_{i+1}^{2}+4 \psi_{i}^{2}+2 \psi_{i-1} \psi_{i+1}-4 \psi_{i} \psi_{i+1}-4 \psi_{i} \psi_{i-1}\right)
\end{aligned}
$$

Then we use the homogeneous boundary conditions Equation (24) and rename the index (increase or decrease the original index by one) in the summations to verify the equivalence relations:

$$
\sum_{i=1}^{M-1} \psi_{i+1}^{2}=\left(\sum_{i=1}^{M-1} \psi_{i}^{2}\right)-\psi_{1}^{2}, \quad \sum_{i=1}^{M-1} \psi_{i-1}^{2}=\left(\sum_{i=1}^{M-1} \psi_{i}^{2}\right)-\psi_{M-1}^{2}
$$

Respectively

$$
\begin{aligned}
\sum_{i=1}^{M-1} \psi_{i-1} \psi_{i+1} & =\sum_{i=0}^{M-2} \psi_{i} \psi_{i+2}=\left(\sum_{i=1}^{M-1} \psi_{i} \psi_{i+2}\right)+\psi_{0} \psi_{2}-\psi_{M-1} \psi_{M+1} \\
& =\left(\sum_{i=1}^{M-1} \psi_{i} \psi_{i+2}\right)-\psi_{M-1}^{2}
\end{aligned}
$$

Similarly we can write Equation (28) as:

$$
\sum_{i=1}^{M-1} \psi_{i-1} \psi_{i+1}=\left(\sum_{i=1}^{M-1} \psi_{i} \psi_{i-2}\right)-\psi_{1}^{2}
$$

Finally we substitute Equations (27)-(29) into Equation (26) to obtain:

$$
h_{x}^{4} \sum_{i=1}^{M-1}\left(\Lambda_{x^{4}} \psi\right)_{i j} \psi_{i j}=h_{x}^{4}\left[\sum_{i=1}^{M-1}\left(\delta_{x}^{+} \delta_{x}^{-} \psi\right)_{i j}^{2}\right]+2 \psi_{1}^{2}+2 \psi_{M-1}^{2}>0
$$

After adding Equation (30) up going through $j$ index from 1 to $N-1$, we complete the proof of the positive definiteness of $\Lambda_{x^{4}}$. Similarly, we can show the theorem about $\Lambda_{y^{4}}$.

(ii) Using the same techniques and going through similar steps, we can verify the following:

$$
\sum_{i=1}^{M-1} \sum_{j=1}^{N-1}\left(\Lambda_{x^{2}} \Lambda_{y^{2}} \psi\right)_{i j} \psi_{i j}=\sum_{i=0}^{M-1} \sum_{j=1}^{N}\left(\delta_{x}^{+} \delta_{y}^{-} \psi\right)_{i j}^{2}>0
$$

Therefore, the proof is completed.

Next, we give some useful theorems about the norm estimates of certain linear operators that appear in the analysis of our difference scheme. Actually, all linear operators in the difference scheme are matrices. For convenience, we use the notations of the difference operators to denote 
the corresponding matrices and denote $E$ as the identity matrix from then on. We define the norm of a matrix $A$ based on the inner product Equation (10) as

$$
\|A\|=\sup _{\psi \neq 0, \psi \in \Phi} \frac{(A \psi, A \psi)^{1 / 2}}{(\psi, \psi)^{1 / 2}}=\max _{(\psi, \psi)^{1 / 2}=1, \psi \in \Phi}(A \psi, A \psi)^{1 / 2}
$$

It should be noted here that the domain in r.h.s is a compact sphere, which allows us to use hereafter max instead of sup in both definitions.

Kellogg's Lemma (Marchuk [25])

For any positive definite matrix $A$ and for any $\sigma>0$ one has

$$
\left\|(E+\sigma A)^{-1}\right\|<1
$$

Now, following Reference [25] we introduce a useful norm $\|\cdot\|_{H}$ defined for any grid function $\psi \in \Phi$ which means $\left(\psi_{i j}\right)$ is defined on the grids Equation (4) and satisfies the boundary conditions Equation (24), namely

$$
\|\psi\|_{H}=\left(h_{x} h_{y} \sum_{i=1}^{M} \sum_{j=1}^{N}\left[\left(\delta_{x}^{-} \psi\right)_{i j}^{2}+\left(\delta_{y}^{-} \psi\right)_{i j}^{2}\right]\right)^{1 / 2}=\left(-\Delta_{h} \psi, \psi\right)^{1 / 2}
$$

Since $\psi$ vanishes on $\partial D$, it is easy to verify that $\|\psi\|_{H}=0$ only when $\psi$ is zero and that some other elementary properties of a norm hold for Equation (34). Similarly, we define the corresponding norm of a matrix $A$ based on this norm as

$$
\|A\|_{H}=\max _{\substack{\psi \neq 0 \\ \psi \in \Phi}} \frac{\|A \psi\|_{H}}{\|\psi\|_{H}}
$$

For convenience, we denote

$$
B \stackrel{\text { def }}{=}-\operatorname{Re}\left(C_{x}^{n+(1 / 2)}+C_{y}^{n+(1 / 2)}\right)+\left(\Lambda_{x^{4}}+\Lambda_{y^{4}}+2 \Lambda_{x^{2}} \Lambda_{y^{2}}\right)
$$

By Theorem 4 and Equation (20) we get that $(B \psi, \psi)>0$ for $\psi \neq 0$, i.e. $B$ is positive definite (or $B>0)$.

The following theorem is one of the key results of the present paper.

\section{Theorem 5}

If $\tau>0$, then $\left\|\left(-\Delta_{h}+(\tau / 2) B\right)^{-1}\left(-\Delta_{h}-(\tau / 2) B\right)\right\|_{H}<1$.

\section{Proof}

Let $\phi=\left(-\Delta_{h}+(\tau / 2) B\right)^{-1}\left(-\Delta_{h}-(\tau / 2) B\right) \psi$ for an arbitrary $\psi \in \Phi$. Then $\phi \in \Phi$, since $\left(-\Delta_{h}+\right.$ $(\tau / 2) B)>0$ is invertible to solve the solution on the interior grid points and we can pose the boundary conditions Equation (24) on $\phi$ freely. so we have

$$
\left(-\Delta_{h}+\frac{\tau}{2} B\right) \phi=\left(-\Delta_{h}-\frac{\tau}{2} B\right) \psi
$$

which can be rewritten as

$$
\left(-\Delta_{h} \psi\right)-\left(-\Delta_{h} \phi\right)=\frac{\tau}{2} B(\psi+\phi)
$$


Taking the inner product with $(\psi+\phi)$ on both sides of Equation (37) and applying Theorem 3 (ensuring that $-\Delta_{h}$ is self-adjoint) we obtain:

$$
\left(-\Delta_{h} \psi, \psi\right)-\left(-\Delta_{h} \phi, \phi\right)=\|\psi\|_{H}^{2}-\|\phi\|_{H}^{2}=\frac{\tau}{2}(B(\psi+\phi),(\psi+\phi))>0
$$

where definition (34) is used for $\|\psi\|_{H}^{2}=\left(-\Delta_{h} \psi, \psi\right)$.

Therefore $\|\psi\|_{H}^{2}>\|\phi\|_{H}^{2}$ and hence $\left\|\left(-\Delta_{h}+(\tau / 2) B\right)^{-1}\left(-\Delta_{h}-(\tau / 2) B\right)\right\|_{H}<1$ which completes the proof.

\section{STABILITY ANALYSIS OF THE FINITE DIFFERENCE SCHEME}

To analyse the stability of our difference scheme Equation (21), we prove the following theorem.

\section{Theorem 6}

If the boundary condition $g(x, t)$ at $y=1$ is assumed to be sufficiently smooth in $[0,1] \times[0, T]$, the difference scheme Equation (21) with Equation (24) is absolutely stable.

Proof

Using previously defined notations we can rewrite Equation (21) as

$$
\left(-\Delta_{h}+\frac{\tau}{2} B\right) \psi^{n+1}=\left(-\Delta_{h}-\frac{\tau}{2} B\right) \psi^{n}-\tau F_{i j}^{n+(1 / 2)}
$$

Then,

$$
\psi^{n+1}=\left(-\Delta_{h}+\frac{\tau}{2} B\right)^{-1}\left(-\Delta_{h}-\frac{\tau}{2} B\right) \psi^{n}-\tau\left(-\Delta_{h}+\frac{\tau}{2} B\right)^{-1} F_{i j}^{n+(1 / 2)}
$$

Here, $F_{i j}^{n+(1 / 2)}$ is given by Equation (23) and we observe that $F_{i j}^{n+(1 / 2)}$ is a vector defined on the interior grid points. Then it is possible to construct a difference analog of the Poisson's equation with $F_{i j}^{n+(1 / 2)}$ playing the role of the nonhomogeneous term in the difference equation, namely

$$
\left(\Delta_{h} W^{n}\right)_{i, j}=F_{i j}^{n+(1 / 2)}
$$

for $i=1, \ldots, M-1$ and $j=1, \ldots, N-1$, with boundary conditions:

$$
W_{0, j}^{n}=W_{M, j}^{n}=0, \quad W_{i, 0}^{n}=W_{i, N}^{n}=0
$$

for $i=1, \ldots, M-1$ and $j=1, \ldots, N-1$.

Since the matrix $\Delta_{h}$ is not singular, there exists a solution $W^{n} \in \Phi$ for Equations (40), (41). Then we rewrite Equation (39) as

$$
\psi^{n+1}=\left(-\Delta_{h}+\frac{\tau}{2} B\right)^{-1}\left(-\Delta_{h}-\frac{\tau}{2} B\right) \psi^{n}+\tau\left[\left(-\Delta_{h}+\frac{\tau}{2} B\right)^{-1}\left(-\Delta_{h}\right)\right] W^{n}
$$

Next, we show $\left\|\left(-\Delta_{h}+(\tau / 2) B\right)^{-1}\left(-\Delta_{h}\right)\right\|_{H} \leqslant 1$. We let nonzero $\phi \in \Phi$ and

$$
\xi=\left[\left(-\Delta_{h}+\frac{\tau}{2} B\right)^{-1}\left(-\Delta_{h}\right)\right] \phi
$$


and then

$$
\left(-\Delta_{h}+\frac{\tau}{2} B\right) \xi=-\Delta_{h} \phi
$$

where we pose the same boundary conditions Equation (24) for $\xi$, since $\left(-\Delta_{h}+(\tau / 2) B\right)>0$ and $-\Delta_{h}$ are invertible under the boundary condition Equation (24). Hence $\xi \in \Phi$ and $\xi$ is nonzero. By Theorem 3, we take the inner product with $\xi$ on both sides of Equation (44) to obtain:

$$
\|\xi\|_{H}^{2}+\frac{\tau}{2}(B \xi, \xi)=h_{x} h_{y} \sum_{i=1}^{M} \sum_{j=1}^{N}\left[\left(\nabla_{i} \xi\right)_{i j}\left(\nabla_{i} \phi\right)_{i j}+\left(\nabla_{j} \xi\right)_{i j}\left(\nabla_{j} \phi\right)_{i j}\right] \leqslant\|\xi\|_{H}\|\phi\|_{H}
$$

where we apply the Cauchy-Schwartz inequality. Since $(B \xi, \xi)>0$, then

$$
\|\xi\|_{H}^{2}<\|\xi\|_{H}\|\phi\|_{H}, \quad\|\xi\|_{H}<\|\phi\|_{H}
$$

which proves that

$$
\left\|\left(-\Delta_{h}+\frac{B}{2}\right)^{-1}\left(-\Delta_{h}\right)\right\|_{H}<1
$$

By Theorem 5 and Equation (42), we obtain:

$$
\begin{aligned}
\left\|\psi^{n+1}\right\|_{H} \leqslant & \left\|\left(-\Delta_{h}+\frac{\tau}{2} B\right)^{-1}\left(-\Delta_{h}-\frac{\tau}{2} B\right)\right\|_{H}\left\|\psi^{n}\right\|_{H} \\
& +\tau\left\|\left(-\Delta_{h}+\frac{\tau}{2} B\right)^{-1}\left(-\Delta_{h}\right)\right\|_{H}\left\|W^{n}\right\|_{H}<\left\|\psi^{n}\right\|_{H}+\tau\left\|W^{n}\right\|_{H}
\end{aligned}
$$

By Equation (34), we have

$$
\left\|W^{n}\right\|_{H}^{2}=\left(-\Delta W^{n}, W^{n}\right)=\left(-F^{n+(1 / 2)}, W^{n}\right) \leqslant\left\|F^{n+(1 / 2)}\right\|\left\|W^{n}\right\|
$$

and since $\Delta_{h}^{-1}$ is a well-defined linear operator, then

$$
\left\|W^{n}\right\| \leqslant\left\|\Delta_{h}^{-1}\right\|\left\|F^{n+(1 / 2)}\right\|
$$

Introducing the last equality in (47) we get

$$
\left\|W^{n}\right\|_{H} \leqslant\left\|\Delta_{h}^{-1}\right\|^{(1 / 2)}\left\|F^{n+(1 / 2)}\right\|
$$

Since the boundary condition $g(x, t)$ is assumed to be sufficiently smooth in $[0,1] \times[0, T]$, then $\left\|F^{n+(1 / 2)}\right\|$ is bounded in $[0, T]$ for each $n$, accordingly. By Equation (48), we can let

$$
\|W\|_{H}=\max _{n}\left\|W^{n}\right\|_{H}
$$

By induction with respect to index $n$ in Equation (45), we finally obtain the inequality

$$
\left\|\psi^{n+1}\right\|_{H}<\left\|\psi^{1}\right\|_{H}+n \tau\|W\|_{H}
$$

where $\psi^{1}$ is the initial condition and $n \tau$ is always less than the length of the time interval for our computation. By Equation (50), we have shown the absolute stability of scheme Equation (21) in the sense of Neumann [25]. 


\section{SPLITTING SCHEME FOR THE INTERNAL ITERATIONS}

To solve the difference equation (21) with Equation (24) directly, one has to invert the matrix representing the $2 \mathrm{D}$ operator $\left(-\Delta_{h}+(\tau / 2) B\right)$. Since the unknown grid function is a vector of dimension $K=(M-1) \times(N-1)$, the size of the matrix is $K \times K$ which renders impractical the straightforward implicit scheme Equation (21). Although the matrix is sparse with only nine nontrivial diagonals, the actual width of the band is $4(N+3)+1$ and that is what matters when treating the system as a system with a banded matrix. Hence, even when using specialized solvers customized for banded matrices, the amount of computations on finer meshes can be staggering. This is the standard case with any fully implicit scheme. A well recognized way to reduce drastically the computations is the operator splitting method.

Direct application of operator splitting is impossible because there is no time derivative of the stream function in the governing equation. This reflects the fact that the $\mathrm{N}-\mathrm{S}$ equations are not Cauchy-Kowalevska system. For this reason we focus on devising an iterative procedure for computing $\psi^{n+1}$ from the fully implicit scheme Equation (21) treating it as a nonlinear elliptic equation for the unknown grid function $\psi^{n+1}$ in which equation the coefficients and the inhomogeneous term are function of the already known grid functions $\psi^{n}$ and $\psi^{n-1}$. In such an approach, the time increment $\tau$ may be considered as a constant coefficient. Respectively all the terms containing of $\psi^{n}$ and the variable coefficients of the operators $C_{x}^{n+(1 / 2)}$ and $C_{y}^{n+(1 / 2)}$ are known. This leads to a 'stationary' problem for solving $\psi^{n+1}$ in each time step. An effective iterative procedure can be devised if an artificial time is added and then an operator splitting technique is employed.

Douglas [15, 16], Peaceman and Rachford [14], and Yanenko [17] presented different iterative processes implementing splitting methods. These have been widely applied to stationary problems in mathematical physics and have proved to be successful in many numerical works. We will follow their ideas (called now 'operator splitting') to construct our scheme in this paper. Specifically Conte and Dames [18] were the first to adapt the Douglas scheme to the case of bi-harmonic operator. The CD scheme exhibits the best of the world of ADI schemes, as being absolutely stable and low cost per iteration, but its rate of convergence has been shown to be rather slow in some cases (see References [28,29]). Recently the present authors contributed a modification of CD scheme that can be radically faster $[30,31]$. The acceleration technique can be used also for the stream function formulation but it goes beyond the scope of the present work which is mostly concerned with the idea of using artificial time and splitting algorithms.

It is important to realize that the original implicit scheme (21) is not amenable to operator splitting. However, when we confine our attention only to the difference scheme Equation (21) for a given time step, that is, the linear system with variable coefficients time-stepping $\psi^{n}$ to $\psi^{n+1}$.

Denote the matrices

$$
\begin{gathered}
A_{x}=-\frac{1}{\tau} \Lambda_{x^{2}}+B_{x}, \quad B_{x}=-\frac{R e}{2} C_{x}^{n+(1 / 2)}+\frac{1}{2} \Lambda_{x^{4}} \\
A_{y}=-\frac{1}{\tau} \Lambda_{y^{2}}+B_{y}, \quad B_{y}=-\frac{R e}{2} C_{y}^{n+(1 / 2)}+\frac{1}{2} \Lambda_{y^{4}} \\
A=A_{x}+A_{y}
\end{gathered}
$$


It is easy to verify that $A_{x}, A_{y}$ and $A$ are positive definite and $\Lambda_{x^{2}} \Lambda_{y^{2}}=\Lambda_{y^{2}} \Lambda_{x^{2}}$ by Equations (5), (6) and (9). Now we write the given vector $G^{n}$ computed by $\psi^{n}$ in Equation (21) as

$$
G^{n}=-F+\left[-\frac{1}{\tau} \Delta_{h}+\frac{R e}{2}\left(C_{x}^{n+(1 / 2)}+C_{y}^{n+(1 / 2)}\right)-\frac{1}{2}\left(\Lambda_{x^{4}}+\Lambda_{y^{4}}+2 \Lambda_{x^{2}} \Lambda_{y^{2}}\right)\right] \psi^{n}
$$

Then we recast Equation (21) into:

$$
\left(A+2 \Lambda_{x^{2}} \Lambda_{y^{2}}\right) \psi^{n+1}=G^{n}
$$

Since the above difference scheme approximates an equation whose highest-order derivatives correspond to the bi-harmonic operator, we can treat our scheme as a scheme for bi-harmonic equation. Note that the matrix $\left(A+2 \Lambda_{x^{2}} \Lambda_{y^{2}}\right)$ is positive definite by Theorem 4 and (36). When $G^{n}$ is given, to solve for the unknown grid function $\psi^{n+1}$ means to invert the $2 \mathrm{D}$ matrix $\left(A+2 \Lambda_{x^{2}} \Lambda_{y^{2}}\right)$ in Equation (55). This is a well-posed stationary problem where $1 / \tau$ in $A$ acts as a given coefficient.

We add to the left-hand side of Equation (55) a derivative $\left(\psi^{n+1, k+1}-\psi^{n+1, k}\right) / \sigma$ with respect to an artificial time with increment (time step) $\sigma$. Here the notation $\psi^{n+1, k}$ denotes the solution of the $k$ th internal iteration from the initial value of the iteration

$$
\psi^{n+1,0} \stackrel{\text { def }}{=} \psi^{n}
$$

Consequently, we replace the original stationary problem by an artificial unsteady problem:

$$
\frac{\psi^{n+1, k+1}-\psi^{n+1, k}}{\sigma}+\left(A+\Lambda_{x^{2}} \Lambda_{y^{2}}\right) \psi^{n+1, k+1}=G^{n}
$$

Since we are interested only in the stationary solution, we need not care for the order of approximation in the artificial time. The main concerns here are the stability and convergence of the iterative scheme. Usually, a first-order in time scheme has better margin of stability when nonlinearity is added and as a rule shows faster convergence. Equation (57) shows how a first-order in time fully implicit scheme for $\psi^{n+1, k+1}$ should look like. We call it 'the scheme in full time steps'. It can be rewritten as follows:

$$
\psi^{n+1, k+1}=\left(E+\sigma\left(A+\Lambda_{x^{2}} \Lambda_{y^{2}}\right)\right)^{-1} \psi^{n+1, k}+\sigma\left(E+\sigma\left(A+\Lambda_{x^{2}} \Lambda_{y^{2}}\right)\right)^{-1} G^{n}
$$

Since $\left\|\left(E+\sigma\left(A+\Lambda_{x^{2}} \Lambda_{y^{2}}\right)\right)^{-1}\right\|<1$ for any $\sigma>0$ by Kellogg's lemma, it is easy to show that Equation (58) is absolutely stable. But, once again, the implementation of this scheme requires inverting a matrix $\left(E+\sigma\left(A+\Lambda_{x^{2}} \Lambda_{y^{2}}\right)\right)$ of size $(M N)^{2}$ with a very large band, so that the iterative scheme Equation (57) is not constructive whereas it is the reliable starting point to construct an economical time-stepping scheme. To this end, we use the classical factorization based on the coordinate splitting of the operator.

To implement the splitting scheme for the internal iterations, we need to deal with the matrix $\Lambda_{x^{2}} \Lambda_{y^{2}}$ approximating the mixed derivative in the equation. Generally, in the splitting scheme for usual nonstationary problems $\Lambda_{x^{2}} \Lambda_{y^{2}}$ is treated explicitly because it cannot be split. But the positivity of $\Lambda_{x^{2}} \Lambda_{y^{2}}$ will enlarge the norm of the transition operator for the splitting scheme when it is treated explicitly and prevent the unconditional stability of the scheme. As it is shown in what follows, the application of splitting can make the approximation of the mixed derivative fully implicit.

Another novel element of the present scheme is the implicit treatment of the nonlinear terms which is a continuation of the ideas of the cited author's works. The trade-off of this approach is 
that the operators $A_{x}$ and $A_{y}$ in our scheme have variable coefficients and do not commute. This requires more efforts in analysing the norm of the transition operator, but the rigorous result about the stability and convergence of the scheme makes it unique and justifies the efforts. The fortunate feature of the $\mathrm{N}-\mathrm{S}$ equations in stream function formulation is that the mixed fourth derivative is a product of the two one-dimensional second-order operators. Then the splitting method offers a unique opportunity to make the approximation of the fourth-order derivative implicit without having to invert the non-definite matrix approximating such a derivative.

The respective coefficients contain the matrices $\Lambda_{x^{2}}$ and $\Lambda_{y^{2}}$ multiplied by ratio $\tau / \sigma$ between the time increment $\tau$ and the artificial time increment $\sigma$ which allows one to adjust the values of these coefficients through a judicious choice of $\sigma$. Particularly, when we set $\sigma=\tau^{2}$, the mixed derivative can be treated totally implicit through the factorization of the matrix $\left(E+\sigma\left(A_{x}+A_{y}\right)+\sigma^{2} A_{x} A_{y}\right)$. This is one of the main new results of the present paper.

Now by this special choice of the artificial time step $\sigma$, we are ready to write down the splitting scheme as follows:

$$
\frac{\psi^{n+1, k+1}-\psi^{n+1, k}}{\sigma}+\left(A_{x}+A_{y}\right) \psi^{n+1, k+1}+\Lambda_{x^{2}} \Lambda_{y^{2}} \psi^{n+1, k+1}+\sigma D\left(\psi^{n+1, k+1}-\psi^{n}\right)=G^{n}
$$

where the matrix $D$ is defined as

$$
\begin{aligned}
D= & A_{x} A_{y}-\frac{1}{\tau^{2}} \Lambda_{x^{2}} \Lambda_{y^{2}}=\left(-\frac{R e}{2} C_{x}^{n+(1 / 2)}+\frac{1}{2} \Lambda_{x^{4}}\right)\left(-\frac{R e}{2} C_{y}^{n+(1 / 2)}+\frac{1}{2} \Lambda_{y^{4}}\right) \\
& +\frac{1}{\tau} \Lambda_{x^{2}}\left(\frac{R e}{2} C_{y}^{n+(1 / 2)}-\frac{1}{2} \Lambda_{y^{4}}\right)+\frac{1}{\tau} \Lambda_{y^{2}}\left(\frac{R e}{2} C_{x}^{n+(1 / 2)}-\frac{1}{2} \Lambda_{x^{4}}\right)
\end{aligned}
$$

It is easy to see that we add a small term $\sigma D\left(\psi^{n, k+1}-\psi^{n}\right)$ on the right-hand side of Equation (58) to obtain Equation (59) and

$$
\left(\sigma D+\Lambda_{x^{2}} \Lambda_{y^{2}}\right) \psi^{n+1, k+1}=\left(\sigma A_{x} A_{y}-\frac{\sigma}{\tau^{2}} \Lambda_{x^{2}} \Lambda_{y^{2}}+\Lambda_{x^{2}} \Lambda_{y^{2}}\right) \psi^{n+1, k+1}=\sigma A_{x} A_{y} \psi^{n+1, k+1}
$$

since $\sigma / \tau^{2}=1$. Then $\sigma$ is multiplied on both sides of Equation (59) and consequently by Equation (61) the final version of our splitting scheme is obtained by the factorization of the matrices on the right-hand side:

$$
\left(E+\sigma A_{x}\right)\left(E+\sigma A_{y}\right) \psi^{n+1, k+1}=\psi^{n+1, k}+\sigma G^{n}+\sigma^{2} D \psi^{n}
$$

Theorem 7

The splitting scheme Equation (62) is absolutely stable.

Proof

We rewrite Equation (62) as

$$
\psi^{n+1, k+1}=\left(E+\sigma A_{y}\right)^{-1}\left(E+\sigma A_{x}\right)^{-1} \psi^{n+1, k}+\sigma\left(E+\sigma A_{y}\right)^{-1}\left(E+\sigma A_{x}\right)^{-1}\left(G^{n}+\sigma D \psi^{n}\right)
$$

Then

$$
\begin{aligned}
\left\|\psi^{n+1, k+1}\right\| \leqslant & \left\|\left(E+\sigma A_{y}\right)^{-1}\right\|\left\|\left(E+\sigma A_{x}\right)^{-1}\right\|\left\|\psi^{n+1, k}\right\| \\
& +\sigma\left\|\left(E+\sigma A_{y}\right)^{-1}\right\|\left\|\left(E+\sigma A_{x}\right)^{-1}\right\|\left\|\left(G^{n}+\sigma D \psi^{n}\right)\right\|
\end{aligned}
$$


Since $A_{x}>0$ and $A_{y}>0$, we have $\left\|\left(E+\sigma A_{y}\right)^{-1}\right\|<1$ and $\left\|\left(E+\sigma A_{x}\right)^{-1}\right\|<1$ by Kellogg's lemma. So

$$
\left\|\psi^{n+1, k+1}\right\|<\left\|\psi^{n+1, k}\right\|+\sigma\left\|\left(G^{n}+\sigma D \psi^{n}\right)\right\|
$$

and hence by the induction of index $k$, we have

$$
\left\|\psi^{n+1, k}\right\|<\left\|\psi^{n+1,0}\right\|+k \sigma\left\|\left(G^{n}+\sigma D \psi^{n+1,0}\right)\right\|
$$

and by definition (56)

$$
\left\|\psi^{n+1, k}\right\|<\left\|\psi^{n}\right\|+k \sigma\left\|\left(G^{n}+\sigma D \psi^{n}\right)\right\|
$$

where $G^{n}$ is considered as a constant grid function in the internal iterations for the $(n+1)$ th full time step. Consequently, Equation (62) is absolutely stable.

\section{Theorem 8}

The internal iterative procedure for the splitting scheme Equation (62) converges, in the sense that $\lim _{k \rightarrow \infty} \psi^{n+1, k}$ exists.

Proof

We write Equation (62) for the $k$ th iteration as

$$
\psi^{n+1, k}=\left(E+\sigma A_{y}\right)^{-1}\left(E+\sigma A_{x}\right)^{-1} \psi^{n+1, k-1}+\sigma\left(E+\sigma A_{y}\right)^{-1}\left(E+\sigma A_{x}\right)^{-1}\left(G^{n}+\sigma D \psi^{n}\right)
$$

Then subtract Equation (65) from Equation (62):

$$
\psi^{n+1, k+1}-\psi^{n+1, k}=\left(E+\sigma A_{y}\right)^{-1}\left(E+\sigma A_{x}\right)^{-1}\left(\psi^{n+1, k}-\psi^{n+1, k-1}\right)
$$

Hence

$$
\begin{aligned}
\left\|\psi^{n+1, k+1}-\psi^{n+1, k}\right\| & \leqslant\left\|\left(E+\sigma A_{y}\right)^{-1}\right\|\left\|\left(E+\sigma A_{x}\right)^{-1}\right\|\left\|\psi^{n+1, k}-\psi^{n+1, k-1}\right\| \\
& <p_{1} p_{2}\left\|\psi^{n+1, k}-\psi^{n+1, k-1}\right\|
\end{aligned}
$$

where $p_{1}$ and $p_{2}$ are constants such that

$$
\left\|\left(E+\sigma A_{y}\right)^{-1}\right\| \leqslant p_{1}<1, \quad\left\|\left(E+\sigma A_{x}\right)^{-1}\right\| \leqslant p_{2}<1
$$

since matrices $A_{x}$ and $A_{y}$ are fixed through all internal iterations for the same $n$. Clearly, Equation (68) implies that $\lim _{k \rightarrow \infty} \psi^{n+1, k}$ exists.

Theorem 6 asserts that the difference scheme Equation (21) is absolutely stable with respect to the real time for any time step $\tau$, and that the solution $\psi^{n+1}$ exists for each time step. Now we prove that the internal iterations can be designed to obtain a solution which approximates the solution $\psi^{n+1}$ from Equation (21) within the order $O\left(\tau^{2}+h_{x}^{2}+h_{y}^{2}\right)$ agreeing with the order by which the finite difference scheme Equation (21) approximates the differential equation. Denote

$$
v^{k}=\psi^{n+1, k}-\psi^{n+1}, \quad v^{0}=\psi^{n}-\psi^{n+1}
$$

then subtract Equation (55) from Equation (59) to have:

$$
\frac{v^{k+1}-v^{k}}{\tau^{2}}+\left(A_{x}+A_{y}+\Lambda_{x^{2}} \Lambda_{y^{2}}\right) v^{k+1}+\tau^{2}\left(A_{x} A_{y}-\frac{\Lambda_{x^{2}} \Lambda_{y^{2}}}{\tau^{2}}\right)\left(v^{k+1}-v^{0}\right)=0
$$


and then multiply Equation (69) by $\tau^{2}$ to complete the factorization:

$$
\left(E+\tau^{2} A_{x}\right)\left(E+\tau^{2} A_{y}\right) v^{k+1}=v^{k}+\tau^{4}\left(A_{x} A_{y}-\frac{\Lambda_{x^{2}} \Lambda_{y^{2}}}{\tau^{2}}\right) v^{0}
$$

Denote now the transition matrix as $T=\left(E+\tau^{2} A_{y}\right)^{-1}\left(E+\tau^{2} A_{x}\right)^{-1}$. Then

$$
v^{k+1}=T v^{k}+\tau^{4} T\left(A_{x} A_{y}-\frac{\Lambda_{x^{2}} \Lambda_{y^{2}}}{\tau^{2}}\right) v^{0}
$$

since we have shown that $\|T\|<p_{1} p_{2}<1$ in Theorem 9. Consequently by induction on index $k$ in Equation (71), we obtain that

$$
v^{k}=T^{k} v^{0}+\tau^{4}\left(T^{k}+T^{k-1}+\cdots+T\right)\left(A_{x} A_{y}-\frac{\Lambda_{x^{2}} \Lambda_{y^{2}}}{\tau^{2}}\right) v^{0}
$$

We first prove the following two theorems.

\section{Theorem 9}

$\|T\| /(1-\|T\|) \approx O(1 / \tau)$ on grid Equation (4) when $\left(\pi h_{x} / 2\right) \ll 1$ and $\left(\pi h_{y} / 2\right) \ll 1$.

Proof

In the beginning we show that

$$
\left\|\left(E+\tau^{2} A_{x}\right)^{-1}\right\|<\left(1+\tau \frac{8}{h_{x}^{2}} \sin ^{2}\left(\frac{\pi h_{x}}{2}\right)\right)^{-1 / 2}
$$

We have Equation (32) for the norm, namely

$$
\left\|\left(E+\tau^{2} A_{x}\right)^{-1}\right\|^{2}=\max _{\substack{\psi \neq 0 \\ \psi \in \Phi}} \frac{\left(\left(E+\tau^{2} A_{x}\right)^{-1} \psi,\left(E+\tau^{2} A_{x}\right)^{-1} \psi\right)}{(\psi, \psi)}
$$

Let us introduce a new grid function

$$
\phi=\left(E+\tau^{2} A_{x}\right)^{-1} \psi
$$

where we can pose the same boundary conditions Equation (24) for $\phi$ such that $\phi \in \Phi$. Then Equation (74) can be rewritten as

$$
\begin{aligned}
\left\|\left(E+\tau^{2} A_{x}\right)^{-1}\right\|^{2} & =\max _{\substack{\phi \neq 0 \\
\phi \in \Phi}} \frac{(\phi, \phi)}{\left(\left(E+\tau^{2} A_{x}\right) \phi,\left(E+\tau^{2} A_{x}\right) \phi\right)} \\
& =\left(\min _{\substack{\phi \neq 0 \\
\phi \in \Phi}}\left[1+2 \tau^{2} \frac{\left(-(1 / \tau) \Lambda_{x^{2}} \phi, \phi\right)}{(\phi, \phi)}+2 \tau^{2} \frac{\left(B_{x} \phi, \phi\right)}{(\phi, \phi)}+\tau^{4} \frac{\left(A_{x} \phi, A_{x} \phi\right)}{(\phi, \phi)}\right]\right)^{-1} \\
& <\left(\min _{\substack{\phi \neq 0 \\
\phi \in \Phi}}\left[1+2 \tau^{2} \frac{\left(-(1 / \tau) \Lambda_{x^{2}} \phi, \phi\right)}{(\phi, \phi)}\right]\right)^{-1} \\
& =\frac{1}{1+2 \tau\left(\left(4 / h_{x}^{2}\right) \sin ^{2}\left(\pi h_{x} / 2\right)\right)}
\end{aligned}
$$


because the smallest eigenvalue of matrix $-\Lambda_{x^{2}}$ defined on the rectangular region $\bar{D}$ is equal to $\left(4 / h_{x}^{2}\right) \sin ^{2}\left(\pi h_{x} / 2\right)$. We make use of notations Equations (51), (52) to get

$$
\left(B_{x} \phi, \phi\right)=-\frac{R e}{2}\left(C_{x}^{n+(1 / 2)} \phi, \phi\right)+\frac{1}{2}\left(\Lambda_{x^{4}} \phi, \phi\right)=0+\frac{1}{2}\left(\Lambda_{x^{4}} \phi, \phi\right)>0
$$

When $\left(\pi h_{x} / 2\right) \ll 1$, we have $\left(4 / h_{x}^{2}\right) \sin ^{2}\left(\pi h_{x} / 2\right) \approx \pi^{2}$ and then approximately

$$
\left\|\left(E+\tau^{2} A_{x}\right)^{-1}\right\|<\left(\frac{1}{1+2 \pi^{2} \tau}\right)^{1 / 2}
$$

Similarly, we can show that when $\left(\pi h_{y} / 2\right) \ll 1$, approximately we have

$$
\left\|\left(E+\tau^{2} A_{y}\right)^{-1}\right\|<\left(\frac{1}{1+2 \pi^{2} \tau}\right)^{1 / 2}
$$

Then

$$
\|T\| \leqslant\left\|\left(E+\tau^{2} A_{y}\right)^{-1}\right\|\left\|\left(E+\tau^{2} A_{y}\right)^{-1}\right\|<\frac{1}{1+2 \pi^{2} \tau}<1
$$

Therefore,

$$
\frac{\|T\|}{1-\|T\|}=\frac{1}{(1 /\|T\|)-1}<\frac{1}{\left(\left(1 / 1+2 \pi^{2} \tau\right)\right)^{-1}-1}=\frac{1}{2 \pi^{2} \tau}
$$

\section{Theorem 10}

If the solution of problem Equation (1) with boundary conditions Equation (2) and initial condition Equation (3) is sufficiently smooth on $D \times[0, T]$, then $\|\left(A_{x} A_{y}-\left(\Lambda_{x^{2}} \Lambda_{y^{2}} / \tau^{2}\right) v^{0} \|=O(1)\right.$.

Proof

Since the analytic solution $\psi$ is assumed to be sufficiently smooth and $\left(A_{x} A_{y}-\left(\Lambda_{x^{2}} \Lambda_{y^{2}} / \tau^{2}\right)\right) v^{0}$ is given by Equation (60), then we can have:

$$
\begin{aligned}
\left(A_{x} A_{y}-\frac{1}{\tau^{2}} \Lambda_{x^{2}} \Lambda_{y^{2}}\right) v^{0}= & \tau\left(\frac{R e}{2} C_{x}^{n+(1 / 2)}-\frac{1}{2} \Lambda_{x^{4}}\right)\left(\frac{R e}{2} C_{y}^{n+(1 / 2)}-\frac{1}{2} \Lambda_{y^{4}}\right)\left(\frac{\psi^{n+1}-\psi^{n}}{\tau}\right) \\
& -\Lambda_{x^{2}}\left(\frac{\operatorname{Re}}{2} C_{y}^{n+(1 / 2)}-\frac{1}{2} \Lambda_{y^{4}}\right)\left(\frac{\psi^{n+1}-\psi^{n}}{\tau}\right) \\
& -\Lambda_{y^{2}}\left(\frac{\operatorname{Re}}{2} C_{x}^{n+(1 / 2)}-\frac{1}{2} \Lambda_{x^{4}}\right)\left(\frac{\psi^{n+1}-\psi^{n}}{\tau}\right) \\
= & \frac{1}{2}\left[\frac{1}{2} \tau\left(-\operatorname{Re} \frac{\partial \Delta \psi}{\partial y} \frac{\partial}{\partial x}-\frac{\partial^{4}}{\partial x^{4}}\right)\left(\operatorname{Re} \frac{\partial \Delta \psi}{\partial x} \frac{\partial}{\partial y}-\frac{\partial^{4}}{\partial y^{4}}\right)\right. \\
& -\frac{\partial^{2}}{\partial x^{2}}\left(\operatorname{Re} \frac{\partial \Delta \psi}{\partial x} \frac{\partial}{\partial y}-\frac{\partial^{4}}{\partial y^{4}}\right)
\end{aligned}
$$




$$
\begin{aligned}
& \left.-\frac{\partial^{2}}{\partial y^{2}}\left(-\operatorname{Re} \frac{\partial \Delta \psi}{\partial y} \frac{\partial}{\partial x}-\frac{\partial^{4}}{\partial x^{4}}\right)\right]\left.\frac{\partial \psi}{\partial t}\right|_{x=i h_{x}, y=j h_{y}, t=(n+(1 / 2)) \tau} \\
& +O\left(h_{x}^{2}+h_{y}^{2}+\tau^{2}\right)=O(1)
\end{aligned}
$$

since the above derivatives are bounded in the compact domain $D \times[0, T]$ under the assumption that the solution $\psi$ is sufficiently smooth.

Making use of Theorems 9 and 10, we show that the following estimate holds for $v^{k}$ from Equation (72):

$$
\left\|v^{k}\right\| \leqslant\|T\|^{k}\left\|v^{0}\right\|+\tau^{4}\left(\frac{\|T\|}{1-\|T\|}\right)\left\|\left(A_{x} A_{y}-\frac{\Lambda_{x^{2}} \Lambda_{y^{2}}}{\tau^{2}}\right) v^{0}\right\|=O\left(\tau^{3}\right)
$$

for sufficiently large $k$.

Note that through out the proof, the grid functions $v^{0}$ and $\psi^{n}$ are taken at the point for $i=1, \ldots, M-1$ and $j=1, \ldots, N-1$, i.e. the following notation is implicit:

$$
v^{0},\left.\psi^{n}\right|_{x=i h_{x}, y=j h_{y}, t=n \tau}
$$

Now we can return to (81) and complete the proof about the consistency of the scheme by noting that since for large $k$ one has $\|T\|^{k} \ll 1$, then one can always find large enough (but reasonably large) $k$ so that $\|T\|^{k} \leqslant \tau^{2}$ which makes the term $\|T\|^{k} v^{0} \sim O\left(\tau^{3}\right)$. This proves the consistency of the scheme in full time steps (physical time). Since this is a very rough estimate, the practical consistency is achieved for much lesser $k$.

\section{COMPUTATIONAL EFFECTIVENESS}

Theorem 9 gives us a rough but useful estimate of the upper-bound of the norm $\|T\|$ so that we can assess the maximal number of the internal iterations required for each full time step (physical time). First we notice that the function $f(x)=(1+(1 / x))^{x}$ is a strictly increasing function on the interval $x \in(0, \infty)$ and $f(2)>2$. Let $K^{*}=1 / 2 \pi^{2} \tau$ and we assume that $K^{*} \geqslant 2$ for a certain sufficiently small $\tau$. By Equation (78) and the definition of $K^{*}$, we have

$$
\|T\|^{k}<\frac{1}{\left[\left(1+\left(1 / K^{*}\right)\right)^{K^{*}}\right]^{k / K^{*}}} \leqslant \frac{1}{[f(2)]^{k / K^{*}}}<\frac{1}{2^{k / K^{*}}} \leqslant \tau^{2}
$$

The last inequality is sufficient to derive the upper-bound $K_{0}$ of the internal iteration number $k$ needed in each physical time step. Then it is easy to show that

$$
\frac{-\ln \tau}{\pi^{2} \tau \ln 2} \leqslant K_{0} \leqslant \frac{-\ln \tau}{\pi^{2} \tau \ln 2}+1
$$

where $K_{0}$ is an integer. If $\tau$ is not very small such that $K^{*}<2$, obviously by Equation (78) we will have smaller $\|T\|$ which gives even faster convergence so that lesser number of internal iterations is needed. Actually it will be just one internal iteration in such a case.

Now we are ready to analyse the computational complexity of our scheme. Since our scheme Equation (21) is absolutely stable, we have the freedom in the choice of $\tau$. It is easy to see 
that $\tau \sim \max \left(h_{x}, h_{y}\right)=1 / \min \{M, N\}$ is a reasonable choice in implementing our scheme which is second-order accurate both in time and space variables. Without loss of generality, we let $M \leqslant N$ and then $\tau=1 / M$. By Equation (83), we have

$$
K_{0} \approx \frac{M \ln M}{\pi^{2} \ln 2}
$$

In each internal iteration, we only need to invert five-diagonal matrices according to the splitting scheme Equation (62) where it takes about $30(M-1)(N-1)$ calculations by using the Gaussian elimination method. Therefore, the total calculations $C_{\text {total }}$ of $K_{0}$ internal iterations for one physical time step are

$$
C_{\text {total }} \approx \frac{30(M-1)(N-1) M \ln M}{\pi^{2} \ln 2} \sim O\left(N M^{2} \ln M\right)
$$

which is much less than the total calculations $O\left([(M-1)(N-1)]^{2}\right)$ needed in inverting directly the $(M-1)(N-1)$ by $(M-1)(N-1)$ full matrix $\left(-\Delta_{h}+(\tau / 2) B\right)$ for the difference equations Equation (21).

Here is to be mentioned that recently we have succeeded to accelerated the rate of convergence of the iterative splitting procedures for bi-harmonic operators in References $[30,31]$ up to five to nine times over the standard rate. Applying one of the procedures developed in those works gives additional edge to the algorithm presented here.

\section{FEATURING EXAMPLE}

As a featuring example for the scheme proposed here we choose the lid-driven cavity flow as one of the most studied benchmark problems. Since the focus of the present work is on the unsteady aspect of the problem, we consider (see Reference [32]) the flow in a square cavity created by the oscillatory motion of the upper lid in its own plane. The motion of the lid is described by its horizontal velocity $U \cos (\omega t)$ where $\omega$ is the frequency. Then the time can be made dimensionless by $\omega^{-1}$ and the dimensionless the $\mathrm{N}-\mathrm{S}$ equations read

$$
\beta \frac{\partial \Delta \psi}{\partial t}+\operatorname{Re}\left(\frac{\partial \psi}{\partial y} \frac{\partial \Delta \psi}{\partial x}-\frac{\partial \psi}{\partial x} \frac{\partial \Delta \psi}{\partial y}\right)-\Delta^{2} \psi=0
$$

where $R e=U L / v$ is the Reynolds number, and $\beta=\omega L^{2} / v$ is the dimensionless frequency parameter. Respectively, the boundary conditions and initial condition are in the same form as Equations (2) and (3) with $g(x, t)=\cos t$. This is discontinuous b.c. for the velocity and the implication of this fact has already been discussed in the end of Section 4.

To demonstrate the performance of the new algorithm, we chose the frequency parameter and the Reynolds number to be of the same order which results into a stronger interaction between the viscous and inertial effects making the flow harder to treat numerically. To be specific, we set $R e=\beta=200$ for the test calculations.

To verify the second order of approximation in space, we consider the solution obtained on grid $160 \times 160$ as the 'exact' solution and compute the $L_{1}$ norm of the difference between the 'exact' solution and the solution on two different coarser grids. Table I presents the result.

In order to verify the second-order approximation in time, we set $M=N=160$ and use three different numbers of time steps within a period. Then for the time increment we have, respectively, 
Table I. Verification of the order of spatial approximation (different norms for the error).

\begin{tabular}{lllc}
\hline Grid size & $L_{\infty}\left(i_{\max }, j_{\max }\right)$ & \multicolumn{1}{c}{$L_{1}$} & $L_{2}$ \\
\hline $40 \times 40$ & $2.262 \mathrm{E}-3(2,39)$ & $2.556 \mathrm{E}-4$ & $4.645 \mathrm{E}-4$ \\
$80 \times 80$ & $6.777 \mathrm{E}-4(2,79)$ & $5.904 \mathrm{E}-5$ & $1.062 \mathrm{E}-4$ \\
Ratio & 3.3378 & 4.392 & 4.3738 \\
\hline
\end{tabular}

Table II. Distance from the reference solution with $\tau=2 \pi / 400$ for two different time increments.

\begin{tabular}{lcc}
\hline Time increment & \multicolumn{1}{c}{$L_{\infty}$} & $L_{2}$ \\
\hline$\tau=2 \pi / 100$ & 0.0468 & 0.0152 \\
$\tau=2 \pi / 200$ & 0.01206 & 0.0042 \\
Ratio & 3.881 & 3.619 \\
\hline
\end{tabular}
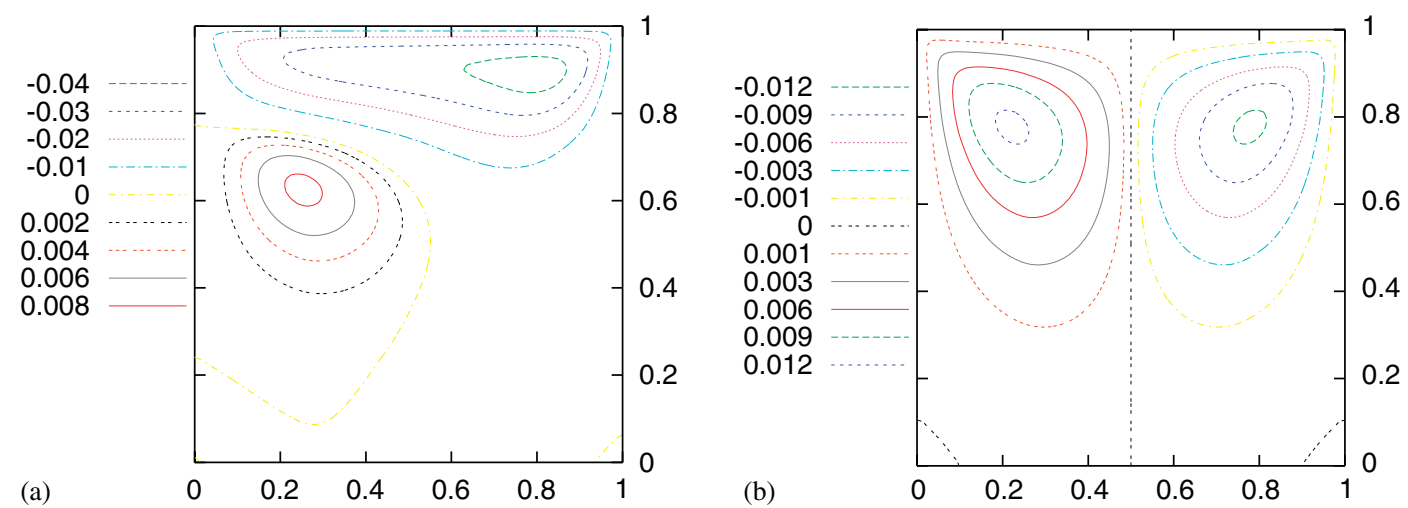

Figure 1. Streamlines of the solution for $\beta=R e=200, \tau=2 \pi / 400$, and $160 \times 160$ grid:

(a) $t=2 \pi$; and (b) steady streaming.

$\tau=2 \pi / 100,2 \pi / 200$, and $2 \pi / 400$. Considering the solution with the smallest time increment as the reference ('exact') solution, we compute the maximum over one time period of the $L_{\infty}$ and $L_{2}$ norms of the difference between the two other solutions and the reference solution. Table II shows that the ratio is close to 4 which means that the order of approximation in time of the scheme is two.

Having an efficient algorithm at one's disposal, one can compute different characteristics of the flow. Since we consider an example of a flow with harmonic oscillations of the boundary, we time stepped the solution until 'convergence' between two consecutive periods is reached in the sense that the solution over the $(k+1)$ th period virtually coincides with the solution over the $k$ th period. Then we compute the functions over one more period beginning with the initial condition that secures the periodicity of the solution. Figure 1(a) gives a snapshot of the stream function 
at the end of a complete period of time. Unlike the steady flow in lid-driven cavity for moderate Reynolds numbers, the unsteady flow consists of two counterrotating (but not symmetric) vortices over the bigger part of the period. We cannot present more detailed investigation of the physical characteristics of the flow here because of the size of the paper and because the present paper is mostly concerned with the development of the algorithm itself.

Another important characteristic of the pulsatile flow is that it has a steady part despite the fact that the boundary condition has strictly oscillatory behaviour with zero mean motion. The steady streaming in oscillatory lows is a well recognized phenomenon that arises due to nonlinearity (see Reference [32] for this particular flow). When computing the last period, we integrate the solution in time for each spatial point and thus we obtain the steady streaming. Figure 1(b), shows our result for the steady streaming. It is in very good quantitative agreement with Reference [32]. The steady flow is symmetric with respect the vertical middle line and exhibits two different vortices. We have computed the flow for $\beta=R e=400$ and in agreement with Reference [32] four different vortices appear for the steady flow.

The results of this section validate the proposed algorithm. The algorithm has already been applied in Reference [33] to a more complicated physical problem involving natural convection in vertical slots, and showed reliable performance.

\section{CONCLUSIONS}

In the present paper, we consider a new scheme for the unsteady Navier-Stokes equations in terms of stream function. We construct an implicit time-stepping scheme and derive the fourthorder elliptic equation for the grid stream function at certain given time stage. We show that this higher-order elliptic problem can be effectively solved numerically by adding an artificial time and constructing of an economical implicit splitting scheme for the artificial parabolic problem.

We propose a special way to approximate the nonlinear advective terms and show that the respective difference operators are antisymmetric (nondissipative). The latter means that there is no second-order artificial viscosity in the scheme. The special treatment of the nonlinear terms allows us to prove that the scheme for the 'full time' steps is absolutely stable and it is of second-order of approximation both in time and space.

The iterative splitting scheme for time-stepping with the artificial time is thoroughly investigated and several important properties are proved, such as absolute stability with respect to the increment of the artificial time. This achievement is possible because we were able to make the approximation of the mixed fourth spatial derivative fully implicit. On the basis of judicious choice of the artificial time increment we prove that the implicit approximation in the internal iteration does not deteriorate the second-order of approximation of the original scheme for the physical time. As a result, a fully implicit unconditionally stable iterative scheme is constructed for the unsteady stream function equation.

The computational effectiveness of the scheme is investigated and it is proved that the number of operations needed to complete a single step of the physical time is $O\left(N M^{2} \ln M\right)$ where $M$ and $N$ are the numbers of grid points in the two spatial directions and $M$ stands for the smaller of them.

A featuring example is elaborated and the practical convergence of the scheme is shown to follow the theoretical predictions. 


\section{REFERENCES}

1. Temam R. Navier-Stokes Equations. North-Holland: Amsterdam, 1977.

2. Mallison GD, de Vahl Davis G. The method of false transients for the solution of coupled elliptic equations. Journal of Computational Physics 1973; 12:435-461.

3. Smagulov Sh, Christov CI. An iterationless implementation of boundary condition for vorticity function. Preprint 20, Inst. Theor. Appl. Mech., Russian Acad. Sci., Novosibirsk, 1980 (in Russian).

4. Quartapelle L. Vorticity conditioning in the computation of two-dimensional viscous flows. Journal of Computational Physics 1981; 40:453-477.

5. Vabishchevich PN. Implicit finite-difference schemes for the nonstationary Navier-Stokes equations with the stream function and vorticity as variables. Differential Equations 1984; 20:820.

6. Churbanov AG, Pavlov AN, Vabishchevich PN. Operator-splitting methods for the incompressible Navier-Stokes equations on non-staggered grids. Part 1: First order schemes. International Journal for Numerical Methods in Fluids 1995; 21:617-640.

7. Karnaidakis G, Israeli M, Orszag SA. High-order splitting methods for the incompressible Navier-Stokes equations. Journal of Computational Physics 1991; 97:414-443.

8. Kim J, Moin P. Application of fractional-step method to incompressible Navier-Stokes equations. Journal of Computational Physics 1985; 59:308-323.

9. Rosenfeld M, Kwak D, Vinokur M. A fractional step solution method for the unsteady incompressible NavierStokes equations in generalized coordinate system. Journal of Computational Physics 1991; 94:102-137.

10. Henshaw AD. A fourth-order accurate method for the incompressible Navier-Stokes equations on overlapping grids. Journal of Computational Physics 1994; 113:13-35.

11. Gresho PhM. Incompressible fluid dynamics: some fundamental formulation issues. Annual Review of Fluid Mechanics 1991; 23:413-453.

12. Burggraf O. Analytical and numerical studies of the structure of steady separated flows. Journal of Fluid Mechanics 1966; 24:113-151.

13. Schreiber R, Keller HB. Driven cavity flows by efficient numerical techniques. Journal of Computational Physics 1983; 49:310-333.

14. Peaceman DW, Rachford Jr HH. The numerical solution of parabolic and elliptic differential equations. SIAM Journal 1953; 3:28-43.

15. Douglas J. On the numerical integration of $\partial^{2} u / \partial x^{2}+\partial^{2} u / \partial y^{2}$ by implicit methods. SIAM Journal 1955; 3:42-65.

16. Douglas J. Alternating-directions methods for three space variables. Numerische Mathematik 1962; 4:41-63.

17. Yanenko NN. Method of Fractional Steps. Gordon and Breach: London, 1971.

18. Conte SD, Dames RT. On an alternating direction method for solving the plate problem with mixed boundary conditions. Journal of the ACM 1960; 7:264-273.

19. Christov CI, Ridha A. Splitting scheme for iterative solution of bi-harmonic equation. Application to 2D NavierStokes problems. In Advances in Numerical Methods and Applications, Dimov I, Sendov Bl, Vasilevskii P (eds). Proceedings of the 3rd International Conference on Numerical Methods $O\left(h^{3}\right)$, August 1994, Sofia, Bulgaria. World Scientific: Singapore, 1994; 341-352.

20. Christov CI, Marinova RS. Implicit vectorial operator splitting for incompressible Navier-Stokes equations in primitive variables. Journal of Computational Technology 2001; 6:93-117.

21. Marinova RS, Christov CI, Marinov TT. A fully coupled solver for incompressible Navier-Stokes equations using operator splitting. International Journal of Computational Fluid Dynamics 2003; 17:371-385.

22. Christov CI, Ridha A. Splitting scheme for the stream-function formulation of $2 \mathrm{D}$ unsteady Navier-Stokes equations. Comptes Rendus de l Academie des Sciences Paris, t. 1995; 320(IIb):441-446.

23. Christov CI, Homsy GM. Nonlinear dynamics of two dimensional convection in a vertically stratified slot with and without gravity modulation. Journal of Fluid Mechanics 2001; 430:335-360.

24. Strikwerda JC. Finite Difference Schemes and Partial Differential Equations, vol. 4. Chapman \& Hall: London, 1989.

25. Marchuk GI. Methods of Numerical Mathematics, vol. 4. Springer: New York, 1975.

26. Arakawa A. Computational design for long-term numerical integration of the equations of fluid motion: twodimensional incompressible flow. Part I. Journal of Computational Physics 1966; 1:119-143.

27. Samarskii AA. The Theory of Difference Schemes. Marcel Dekker: New York, 2001.

28. Greenspan D, Schultz D. Fast finite-difference solution of biharmonic problems. Communications of ACM 1972; 15:347-350. 
29. Ehrlich LW, Gupta MM. Some difference schemes for the biharmonic equation. SIAM Journal on Numerical Analysis 1975; 12:773-790.

30. Tang X-H, Christov CI. An operator splitting scheme for biharmonic equation with accelerated convergence. In Large Scale Scientific Computing, Wasniewski J, Lirkov I, Margenov S (eds), vol. 3743. Springer: New York, 2005; 380-387.

31. Tang X-H, Christov CI. A multiunit adi scheme for biharmonic equation with accelerated convergence. International Journal of Computational Science and Engineering (IJCSE) 2005, accepted.

32. Duck PW. Oscillatory flow inside a square cavity. Journal of Fluid Mechanics 1982; 122:215-234.

33. Tang X-H, Christov CI. Nonlinear waves of the steady natural convection in a vertical fluid layer: a numerical approach. Special Issue of the Journal Mathematics and Computers in Simulation on 'Nonlinear Waves: Computation and Theory' 2005, submitted. 